\title{
REPRESENTACIÓN DEL TEATRO CÓMICO DE PÍO BAROJA: EL HORROROSO CRIMEN DE PEÑARANDA DEL CAMPO
}

\author{
Francisco GutiérRez CARbajo \\ U.N.E.D.- Madrid
}

La producción teatral ha sido hasta hace pocos años uno de los aspectos más desatendidos de la inmensa obra de Pío Baroja, aunque el autor cultivó el género escénico, fue crítico teatral e intervino como actor en alguna ocasión. El reputado novelista escribió obras teatrales como El horroroso crimen de Peñaranda del Campo, Adiós a la bohemia, Arlequín, mancebo de botica, Chinchín, Comediante, Las noches del Café de Alzate y Todo va bien... a veces; ejerció la crítica teatral en el periódico El Globo durante los años 1902 y 1903, como nos ha testimoniado Azorín e interpretó alguno de los papeles de sus obras, que se representaron en el teatro de cámara El Mirlo Blanco, instalado en la casa de los Baroja, en la calle Álvarez Mendizábal de Madrid. Esta producción y actividad escénicas de Pío Baroja sólo en los últimos tiempos han merecido importantes estudios. Se han analizado, así, «la posición barojiana ante el teatro» (AAVV, 1972: 30-35 y Vila Selma, 1956:6-7), su condición de actor (Fernández Almagro, 1954: 36) y las representaciones en El Mirlo Blanco (Hormigón, 1972: 349-355; Rey Faraldos, 1985: 117-127), la «curiosidad» de la dramaturgia barojiana (Chalebois, 1987), «su original aportación al teatro musical español» (Plaza, 1983) y otras cuestiones en torno al arte dramático de nuestro autor (Franco, 1975 y Bloom, 1988).

Sin embargo, la recuperación pública de su producción escénica y de su entraña cómica no se logra hasta el año 1978 con la representación del El horroroso crimen de Peñaranda del Campo en la Sala Cadalso de Ma- 
drid por el Teatro Libre bajo la dirección de José Luis Alonso de Santos. A raíz de esa representación se publican artículos y estudios como los de Ángel Fernández Santos (1978), Enrique Llovet (1979), Josep María Llerena (1980), José Monleón (1980) y Marga Piñero (2005: 187-191), se reeditan varias de sus obras dramáticas (Rubio Jiménez, 1998; Gago Rodó, 1997) y algunos críticos eligen para sus artículos títulos como el siguiente: «Pío Baroja, al fin en los escenarios» (Ynduráin, 1978).

Pío Baroja calificó El horroroso crimen de Peñaranda del Campo de «farsa villanesca», y a esa modalidad teatral y a otras próximas se viene adscribiendo esta pieza. Para Domingo Ynduráin (1978), pertenece a lo que denomina género menor del teatro, en cuanto se sitúa en la tradición formada por los entremeses, bailes, sainetes, y subraya que el tono preciso de la obra es el de la farsa. Enrique Llovet (1979) la incardina en la gran tradición de las «minoridades» dramáticas: bailes, sainetes, jácaras, entremeses, plástica de los famosos pliegos acordelados, centón de cartelones populares, composición de ferias y retórica de mascaradas. Atendiendo al fondo y a la forma de estos «relatos» populares, a la materia general utilizada y al público al que iban destinados, se nutre -según Llovet- de los ingredientes del esperpento. José Monleón (1980: 21) no duda en calificarla de «interesante folletín frustrado», mientras que Ángel Fernández Santos (1980: 28) la encuentra difícilmente encasillable, ya que es una farsa, que de repente deja de ser farsa, es un esperpento y no es un esperpento. Para Jesús Rubio Jiménez (1998: 55) se trata de «una parodia de la literatura truculenta y sanguinaria, que inundaba en aquellos años librerías, quioscos y escenarios...». Más tarde insiste en que «debe ser considerada como una farsa granguiñolesca» (Rubio Jiménez, 1998: 62). Josep $\mathrm{M}^{\mathrm{a}}$ Llerena (1980) encuentra en la obra «un retrato real», «un espectáculo de la crueldad» en la línea de Artaud, «una radiografía grotesca de la realidad» y Marga Piñero (2004: 41) añade que Alonso de Santos eleva «esa radiografía grotesca de la realidad a una obra de caracteres».

Por nuestra parte subrayamos el concepto de farsa, propuesto por Pío Baroja (1948: 1257-1258), que enlaza con la rica tradición del carnaval y con el género cultivado ya «desde Plauto y Terencio hasta nuestros saineteros». La «farsa» sería la forma nuclear del teatro carnavalesco según Huerta Calvo (2001:22), para quien no resulta «exagerado comenzar el itinerario histórico del teatro breve» con la Égloga de Antruejo (1496) y el Auto de Repelón (1509) de Juan del Encina. Con estos antecedentes, y con el fin de contextualizar la obra barojiana en el universo del arte dramático, parece pertinente realizar una breve síntesis histórica de estas formas de teatro breve (farsas, entremeses, sainetes...) a las que hemos prestado atención en otros trabajos (Gutiérrez Carbajo, 2006). 
Lope de Rueda, que une en su persona la condición de autor y comediante, consolidaría, como atestigua Rojas Villandrando en su Viaje entretenido, el género del entremés, aunque él no emplease este nombre. Eugenio Asensio (1971: 13) propone la tesis de la obra corta como «esqueje desgajado de la comedia por Lope de Rueda», y reconoce la matriz festiva del entremés, el sentido de farsa y de carnavalización, aludidos. En esta línea, aparecían superadas las tesis sobre la raíz común alegórico-religiosa del auto y del entremés (Jack, 1923: 15) y asumidos los tipos y mecanismos humorísticos descritos por H. Heidenreich (1962). Estos mecanismos del humor tienen en la Jocoseria de Luis Quiñones de Benavente magníficos ejemplos del «arte nuevo de hacer entremeses». Para Eugenio Asensio quedaba pendiente de investigación la producción dramática corta a partir de 1640, fecha en que puede decirse que Quiñones de Benavente perfila definitivamente el modelo de entremés (Rodríguez-Tordera, 1990: 14). Reconociendo la importancia de los trabajos de Cotarelo y Mori (1911) y de Asensio (1971), los profesores Evangelina Rodríguez y Antonio Tordera (1990: 12) consideran que las obras dramáticas breves del siglo XVII no han gozado de la misma consideración bibliográfica que otros aspectos del arte escénico barroco. Sin embargo, la situación ha ido cambiando y hoy ya contamos con los trabajos de Agustín de la Granja (1981 y 1994), María Luisa Lobato (De la Granja-Lobato, 1999) Evangelina Rodríguez y Antonio Tordera (1990), Javier Huerta Calvo (1996, 1999, 2000 y 2004), Medardo Fraile (1989), Margot Versteeg (2000, 2001), Emilio Javier Peral Vega (2001) y Virtudes Serrano (2004), entre otros.

A partir del siglo XVII el teatro breve se centra especialmente en los sectores populares y marginales de la sociedad, tendencia acentuada en el XVIII con Ramón de la Cruz. Junto al sainete encontramos otras representaciones de teatro breve en la segunda mitad del siglo XVIII, como la zarzuela, el melólogo, la tonadilla y el melodrama. Cuando a principios del siglo XIX la situación política cambia, el teatro menor se politiza. RaffiBéroud (2001: 65) ha estudiado este «teatro urgente», examinando cómo lleva el sello de los acontecimientos: unas veces elogiando el patriotismo de los españoles, otras zahiriendo al invasor y otras incluso ensalzando los beneficios que la dominación francesa podría reportar al país. Durante el último tercio del XIX, el sainete, y sus géneros fronterizos reciben un impulso extraordinario con el florecimiento de los teatros por horas. Como repertorios de estos teatros 'a real' el género chico (con sus sainetes, zarzuelillas, revistas y juguetes...) alcanza una gran repercusión en la vida escénica (Versteeg, 2001: 75-84). El primer tercio del siglo $\mathrm{xX}$, por su parte, conoció una regeneración teatral, surgida de una readaptación de la commedia dell arte y de las formas breves del teatro clásico español. Con 
ellas entroncan jAdiós a la bohemia! y El horroroso crimen de Peñaranda del Campo, de Pío Baroja.

La dramaturgia menor producida entre la última década del siglo XIX y el año 1939 la analiza Emilio Javier Peral en Formas del teatro breve español en el siglo XX (1892-1939), y el año 1940 es el punto de partida que toma Medardo Fraile (1989) para su estudio y antología Teatro español en un acto (1940-1952). Por lo que se refiere a las últimas manifestaciones del siglo xx, Virtudes Serrano (2004) en Teatro breve entre dos siglos recoge y examina veintiún textos de otros tantos autores de edades y estilos muy diversos. Por nuestra parte hemos analizado muestras muy significativas del teatro breve de principios del siglo XXI, con especial referencia a la obra de José Luis Alonso de Santos (Gutiérrez Carbajo, 2006 a y 2006 b).

José Luis Alonso de Santos fue precisamente el encargado de poner en escena El horroroso crimen de Peñaranda del Campo en la Sala Cadalso de Madrid, a finales de 1978. La obra barojiana ya había sido estrenada en 1973 por el Pequeño Teatro de Barcelona, dirigido por $\mathrm{M}^{\mathrm{a}}$ Luisa Oliveda, según reseña de Xavier Fábregas y nota de Andrés Franco, reproducidas por Rubio Jiménez (1998: 62). José Monleón da cuenta en Triunfo $(559,16-$ VI-1973) de un intento fallido de estrenar la obra y Marga Piñero (2005: 188) nos informa de una representación semiprivada por el TEU de Murcia. Sin embargo, la obra alcanza la difusión y recepción merecidas gracias al citado estreno de 1978. Alonso de Santos (1980: 39) ha contabilizado unas cuatrocientas representaciones durante los tres años que estuvo la obra en cartel, en un itinerario que la llevó de la mencionada sala Cadalso al Valencia Cinema, a la sala Villarroel de Barcelona, al Auditórium de Palma de Mallorca, al teatro Lope de Vega de Sevilla y a otros escenarios españoles y extranjeros. Recibió el Premio Ciudad de Segovia y representó a España en el Festival de Palermo.

La farsa de Pío Baroja, en la representación del Teatro Libre, participa del folletín y de la aleluya: del folletín, por lo trágico, y de la aleluya, por lo cómico. La aleluya, sobre la estructura del romance de ciego, provoca los primeros momentos cómicos de la representación, con alusiones al cine y al procedimiento que se está empleando para narrar los hechos.

No se ha prestado atención al hecho de que la versión de Alonso de Santos convierte la farsa o el género "gólfico castellano» en un juego escénico con fuertes elementos andaluces. La música, y en concreto el cante flamenco de Camarón de Isla, contribuyó con fuerza a este giro estilístico. Sobre el director castellano debieron de pesar la naturaleza andaluza de algunos de los principales intérpretes de la obra, como Margarita Piñero o Rafael Álvarez, El Brujo. 
La adaptación acentúa en las primeras representaciones la condena de la pena de muerte sobre la que se sustenta, a pesar del carácter de farsa, uno de los núcleos estructuradores de la obra. El propio autor nos ha proporcionado algunas pistas sobre el horror de estas ejecuciones en Los verdugos: «Una vez, de niño contemplé el paso de un reo que llevaban a ejecutar en la Vuelta del Castillo. Iba en un carrito rodeado de cuatro o cinco curas. Vestía un sayo amarillo pintado con llamas rojas y un birrete. Se llamaba Toribio Eguía. Había matado en Aoiz a un cura y a su sobrina. Dos largas filas de disciplinados encapuchados, con sus cirios amarillos, cantando responsos y letanías, iban delante del carro. Detrás marchaba el verdugo a pie, braceando. Todas las campanas de las iglesias del pueblo tocaban a muerto» (Llerena, 1980: 35). La estructura del montaje contra la pena de muerte, en una sociedad que la tenía establecida como legal, tuvo que modificarse, según explica Alonso de Santos (1980: 40), cuando afortunadamente ésta fue abolida en España en plena época de representaciones. Ello les ayudó a descubrir que la pena de muerte no era el problema básico del texto barojiano, aunque el adaptador sigue dudando de que el autor hubiera escrito esta obra de no haber estado vigente en su tiempo y de no haber quedado impresionado por la ejecución que presenció.

Las declaraciones de Pío Baroja, que tanto se rió de las «fidelidades al texto», les ayudaron también al director y a los actores para obrar con libertad en la recreación de la obra, aunque las modificaciones que introducen no son sustanciales.

En el trabajo de adaptación y de interpretación se operan dos procesos sólo aparentemente antitéticos: por un parte, han de realizar una labor de ampliación, han de transformar una pieza corta pensada para una duración de media hora escasa en un espectáculo de hora y media, el tiempo canónico de la mayoría de las representaciones; por otra, han de llevar a cabo un proceso de síntesis, de condensación. Esta segunda operación se traduce en una reducción e incluso en una supresión de alguno de sus protagonistas. El personaje Martínez, por ejemplo, no aparece en la representación. En el texto de Baroja, este sujeto está explicitando constantemente lo que pasa en la obra y emitiendo sus puntos de vista. Para el director, Martínez hace evidente lo evidente y se les convertía en un personaje redundante e innecesario; de ahí que decidieran prescindir del mismo. Los hermanos de Paz y Caridad quedan reducidos a tres; los personajes del pueblo a siete. Todos los actores tuvieron que hacer al menos el papel de dos personajes, e incluso algunos de tres, como Héctor Roskin que interpretó los papeles del chulo, el Padre Ratera y el verdugo, y Rafael Álvarez, El Brujo que hizo de gitano, guardia y reo. 
El adaptador concede más relieve a algunos personajes como el fotógrafo y el reo. El fotógrafo, en la versión del Teatro Libre, abría y cerraba la representación, y encarnaba el punto de vista del grupo y de su director sobre lo que estaba sucediendo en escena.

El peso del montaje, como ha declarado el propio Alonso de Santos, se centraba en el personaje del reo. En el primer cuadro de la obra y en las primeras escenas de la representación el reo no está presente, pero se hacen referencias constantes a él, y tanto en el texto como en la representación se sigue una sabia estrategia a la hora de suministrar la información, creando la imagen de un ser terrorífico y conflictivo, conquistador y mujeriego y generando un indudable suspense. En el segundo cuadro, o en la segunda escena, aparece ya el reo en la capilla de la cárcel de Peñaranda, con los hermanos de Paz y Caridad, el director de la prisión y el cura. En el cuadro de Baroja está también presente el Padre Ratera, que en la representación ha desaparecido. El autor lo caracteriza como un «joven moreno, con los ojos brillantes». En la representación se acentúa su carácter de antihéroe, de marginal, que para ser algo en la vida se declara culpable de una muerte que no ha cometido, una muerte, que, como tendremos ocasión de contemplar más tarde, no ha cometido nadie. En este personaje centra el director la mayor fuerza y a la vez la mayor vis cómica de la obra. En la representación se subraya ese intento desesperado de Canelo de ser un auténtico protagonista, de desempeñar un papel en la vida, aunque sea el de un condenado a muerte.

El contrapunto de Canelo, o, si se quiere, el antihéroe, viene representado por el verdugo, por el tío Lezna, que Baroja lo describe como «un hombrecito de cincuenta años, muy flaco»», vestido de pana, con una gorrita en la mano, y que, como se observa en la propia acotación entra en escena «temblando». Una de las secuencias con mayor fuerza dramática es el encuentro entre estos dos personajes. El tío Lezna empieza pidiéndole perdón al reo. Canelo no se podía imaginar que este hombre, natural de su propio pueblo, vaya a ser su verdugo y no duda en decirle: « Menudo oficio que ha cogido usté, gachó! ¡Vaya un carguito!». La representación del Teatro Libre introduce unas pequeñas modificaciones en la respuesta del tío Lezna y en el diálogo posterior, suprimiendo términos del texto como «azogado». El propio reo ha de animar al verdugo, y en la representación se incluye un abrazo entre ambos y un pequeño parlamento del tío Lezna, quedando el diálogo así:

EL CANELO. - Me va usté hacer sufrir mucho. Va usté a hacer un serrallo conmigo, tio Lezna.

EL TÍO LEZNA. - No tengas cuidado. Eso, no. Yo he sido siempre hombre de conciencia. 
EL CANELO. - Sí, pero la conciencia de un verdugo no le tranquiliza a uno.

EL TÍO LEZNA. - ¡Maldita sea la pena!. No tengas cuidado, no. Así que ¿me perdonas?

EL CANELO. - Sí, hombre sí. Vaya usté tranquilo (Abrazo).

EL TÍO LEZNA. - Gracias, hijo. Gracias.

La confesión de inocencia que le hace El Canelo a la Marquesa y el telegrama del Jefe de la Policia de Lisboa notificando que «la Sinforosa López» se encuentra allí y en buen estado de salud motivan que se suspenda la ejecución y que El Canelo quede en libertad.

En la tercera parte de la representación, que reproduce el cuadro III de la obra, se nos presenta al tío Lezna y al Canelo en la plaza de Peñaranda, añorando aquellos terribles papeles de reo y de verdugo, aquellos días en los que, al menos, eran algo en la vida. No piensan que estén ya en situación de volver a desempeñar esas funciones, ni de profesar como frailes, ni de inscribirse en el Tercio. Lo más razonable es irse a un bar, al «tupi del Cigüeño», y tomarse «una raja de bacalao, un pedazo de pan y un vasazo de peleón».

Pío Baroja añade un epílogo a la obra, situado en el casino de Peñaranda, donde don Severo, don Zenón, Martínez y Ballenilla -el profesor de Retórica-, mientras juegan al dominó, comentan la obra, «el sainete de ayer noche». El grupo Teatro Libre también reelabora este final, suprimiendo algunas canciones e intentando siempre «convertir lo establecido en ridículo, lo lúgubre en grotesco» (Alonso de Santos, 1980: 44).

El propio director se ha encargado de explicar la representación estrenada en la sala Cadalso, que recorrió numerosos escenarios. Como se ha apuntado, el montaje se realizó, partiendo del folletín y de las formas gráficas de elementos de viñetas, de aleluyas, de composiciones planas. Las secuencias, con unidad en sí mismas, estaban, sin embargo, articuladas entre sí. Buscadas y encontradas las fuerzas en pugna y la línea básica de los conflictos, se cuidó especialmente el proceso que explica el paso del actor al personaje. Se prestó especial atención a la voz, más que en el estreno, en los montajes sucesivos: «En una etapa posterior encontramos las voces de los personajes (voces surgidas ya de la incorporación orgánica del personaje al actor). Fue el paso definitivo y esencial en todo el proceso» (Alonso de Santos, 1980: 45-46).

La escenografía, de la que fue responsable Marco Herreros, así como el sonido y la música, a cargo de Francis Maniglia, «salieron de los ensayos, en un proceso de NECESIDAD». Este mismo proceso siguió el resto de los elementos plásticos y sonoros de la obra. 
Todo ello contribuyó a la magnífica recepción de El horroroso crimen de Peñaranda del Campo y a despertar el interés por la producción escénica de Baroja, cuya tardía valoración no ha debido de ser ajena a la «escasa atención que en el ámbito académico se ha prestado a los géneros literarios, teatrales y cinematográficos donde lo cómico o lo humorístico están presentes» (Ríos Carratalá, 2005: 15).

El humor, la risa, y el resto de las modalidades de lo cómico reciben una valoración muy positiva en la época en la que Pío Baroja escribe El horroroso crimen de Peñaranda del Campo. Ortega y Gasset sitúa la «ironía esencial» en el corazón del «arte nuevo» y para Ramón Gómez de la Serna, el humor no es sólo una modalidad literaria sino también un género de vida. Ramón en Gravedad e importancia del humorismo asegura que este fenómeno «inunda la vida literaria, domina casi todos los estilos» (Gómez de la Serna, 1930: 348). En el estudio del humor, Ramón realiza un recorrido que comprende desde las teorías humorales de Hipócrates y Práxagoras hasta las concepciones endocrínicas y metabólicas de Gregorio Marañón, pasando por filósofos, escritores y pintores como Sócrates, Séneca, Bergson, Spencer, Kant, Arquíloco, Juvenal, el Arcipreste de Hita, la Celestina, Cervantes, Gracián, Quevedo, Góngora, Lafontaine, Bernard Shaw, Oscar Wilde, Valle-Inclán, Goya y Picasso. El humor es, según Ramón, «la actitud más cierta ante la efimeridad de la vida, el deber racional más indispensable, y en su almohada de trivilialidades, mezcladas de gravedades, se descansa con plenitud» (Gómez de la Serna, 1930: 350 ). Con una actitud que recuerda a la de Nietzsche, Ramón observa que el humorismo es lo más limpio de intenciones, de efectismo y de trucos, «es una obligación de alta mar en los siglos, una condición de superioridad». Con una mezcla de greguería y de esperpento, según Juan Chabás (1967), abordó Antonio Espina el humor en Lo cómico contemporáneo (1928), y en El humorismo como evasiva defiende un humor que no sea un escape, un refugio, un burladero, utilizando un símil taurino de Ortega. El concepto de humor en Espina es perfectamente aplicable a la obra estudiada de Pío Baroja, ya que el tratamiento de este recurso implica superar en la escritura la trasposición directa de la realidad, subraya el potencial reflexivo de la ficción, y comporta la idea de que la literatura y el teatro no dejan de ser un espejo, pero se trata no del «espejo a lo largo del camino» sino del que modifica y transfigura la representación.

Pío Baroja en La caverna del humorismo (1919) contrapone este recurso a la retórica, y señala, entre otras cosas, que si la retórica se identifica con la tradición, con la afirmación de valores viejos, el humorismo es invención, es afirmación de valores nuevos. La retórica, como arte de la ornamentación, «necesita masas y líneas fijas, necesita sustancias duras, 
envejecidas por el tiempo, el humorismo es la fantasmagoría de los líquidos y de los gases espirituales. La retórica descansa sobre lo que parece más seguro y respetable, el humorismo en lo que se considera más movedizo y pasajero. La retórica tiende a forzar la armonía de las cosas y a inmovilizar, por tanto, el mundo espiritual; el humorismo tiende a relajar, a dar a todo flexibilidad y blandura» (Baroja, 1986). Baroja aborrece, por una parte, la retórica altisonante, campanuda y hueca, y por otra, la vulgar y casera, presente en algunos autores que no merecen la mejor de sus consideraciones. Así, en el artículo publicado con motivo del estreno de Adiós a la bohemia rechaza la que denomina retórica vulgar, casera, falsamente natural, y argumenta que no puede soportar «la que se encuentra en la fraseología de Galdós, de Dicenta, de Benavente y de Martínez Sierra» (Monleón, 1980: 26). Subrayando estos mismos principios, en el prólogo de El horroroso crimen de Peñaranda del Campo aclara que hay que colgar en «las perchas del guardarropa las prendas de la retórica altisonante». En este contexto su obra se definiría por el rechazo de los valores viejos, de lo seguro y de lo «respetable», de lo inflexible y de lo duro. Con una simple intención higiénica, quiere convertir lo lúgubre en grotesco y llevar las escenas de un tablado a otro tablado. A pesar de sus ataques a la retórica, desde nuestra perspectiva, el humor, tanto en el texto barojiano como en la puesta en escena del mismo por el Grupo Teatro Libre de Madrid, funciona como un importante recurso retórico. Un recurso que, en el sentido amplio en que nosotros lo estudiamos, se relaciona con la ironía, la sátira, la parodia y la crítica. Como señalaría Bajtín (1986:70), este tipo de discurso es muy variado. En un sentido semejante se manifiestan los investigadores actuales de la poética y de la retórica de esta modalidad literaria, como Booth, Jameson (1989 a y b), Linda Hutcheon y Pierre Schoentjes (2003). 


\section{BIBLIOGRAFÍA}

AAVV. 1972. «Sobre la posición barojiana ante el teatro. Encuesta», Primer Acto, 143 (abril, 1972), pp. 30-35.

Alonso De SANTOS, José Luis. 1980. «Nuestro montaje de El horroroso crimen de Peñaranda del Campo de Pío Baroja por el «Teatro Libre» de Madrid, en Pío Baroja, El horroroso crimen de Peñaranda del Campo, Madrid, Vox, 1980, pp. 39-46.

- 1988. "Orientaciones para el montaje», en Pío Baroja, iAdiós a la bohemia!, Arlequín, mancebo de botica y El horroroso crimen de Peñaranda del Campo, Madrid, Biblioteca Nueva-Fundación Argentaria, Int. Jesús Rubio Jiménez, pp. 157-165.

AsEnsio, Eugenio. 1971. Itinerario del entremés: desde Lope de Rueda a Quiñones de Benavente: con cinco entremeses de D. Francisco de Quevedo, Madrid, Gredos, $1971,2^{\mathrm{a}}$ ed. rev.

Baroja, Pío. 1948. El horroroso crimen de Peñaranda del Campo, en OO.CC., Madrid, Biblioteca Nueva, 1948, vol. VI.

- 1980. El horroroso crimen de Peñaranda del Campo, Madrid, Vox.

- 1986. La caverna del humorismo, Madrid, Caro Raggio.

BLoom, Leonard. 1988. «En torno al teatro barojiano», Journal of Society of Basque Studies in America, 8 (1988), pp. 85-90.

Bobes, Ma del Carmen.1987. Semiología de la obra dramática, Madrid,

Taurus.

Canavaggio, Jean.1989. «Sevilla y el teatro a fines del siglo xvI: apostillas a un documento poco conocido». En RUANO DE LA HAZA, José María (ed.).1989. El mundo del teatro español en su Siglo de Oro: ensayos dedicados a John E. Varey, Oawa, Dovehouse Ed.

Cotarelo y Mori, Emilio.1911. Colección de Entremeses, Loas, Bailes, Jácaras y Mojigangas desde fines del siglo XVI a mediados del XVIII, Madrid, BAE, t. 17-18.

ChabÁs, Juan. 1967. Historia de la literatura española. La Habana, Inst. del Libro.

Charlobois, Lucile C. 1987. «El teatro de Pío Baroja: una curiosidad», Nueva Revista de Filología Hispánica, XXXV (1987), pp. 171195.

De la Granja, Agustín. 1981. Entremeses y mojigangas de Calderón para sus autos sacramentales, Granada, Universidad de Granada.

- 1994. «El entremés: la larga risa de un teatro breve». En ARELLANO, Ignacio, GARCÍA RUIZ, Víctor y VITSE, Marc (eds.) 1994. Del horror a la risa. Los géneros dramáticos clásicos. Homenaje a Christiane Faliu-Lacourt, Kassel, Reichenberger, 161-189. 
- y LOBATO, M. L. 1999. Bibliografia descriptiva del teatro breve español (siglos $X V-X X)$. Madrid: Universidad de Navarra/ Iberoamericana/ Vervuert.

Eco, Umberto. 1992. Los límites de la interpretación, Barcelona, Lumen. EspinA, Antonio. 1928. «Lo cómico contemporáneo», El Sol, 24-XII1938.

- «El humorismo como evasiva», El Sol, 13-III-1930.

Fernández, José Ramón. 2003. Pról. a Alonso de Santos. Que siga la comedia. Texto íntegro de La comedia de Carla y Luisa, Madrid, Centro Cultural de la Villa.

Fernández Almagro, Melchor. 1954. «Baroja actor», Índice de Artes y Letras, $70-71$ (enero-febrero, 1954), p. 36.

Fermández-Santos, Ángel. 1980. «Gente libre para un drama libre»y «Pío Baroja, al tablado. Teatro Libre, nómada con El horroroso crimen», en Pío Baroja, El horroroso crimen de Peñaranda del Campo, Madrid, Vox, 1980, pp. 28-30 y 31-32.

Fraile, Medardo (ed.) (1989). Teatro español en un acto, Madrid, Cátedra.

Franco, Andrés. 1975. «El teatro de Baroja», Cuadernos Hispanoamericanos, 296 (febrero, 1975), pp. 277-299.

Gago Rodó, Antonio. 1997. Edición de la ópera chica de Pío Baroja, ¡Adiós a la bohemia, Madrid, U.A.M..

Gómez DE LA SERnA, Ramón.1930. «Gravedad e importancia del humorismo», Revista de Occidente, T. XXVIII (abril-mayo-junio, 1930), pp. 348-368.

Gutiérrez Carbajo, Francisco. 1993. Cine y literatura, Madrid, UNED.

- 2001. "Versiones fílmicas de Bajarse al moro, de José Luis Alonso de Santos y de La mirada del hombre oscuro, de Ignacio del Moral, Anales de Literatura española Contemporánea. «Teatro y Cine», 26, I (2001), 213-237.

- 2003. Int. a Alonso de Santos. Que siga la comedia. Texto íntegro de «La comedia de Carla y Luisa». Madrid, Centro Cultural de la Villa, 5-16.

- 2004. «Carlos Molinero y su interpretación fílmica de la obra teatral Salvajes, de José Luis Alonso de Santos». Signa, 13, 173-184.

- 2006 a, ed. José Luis Alonso de Santos, Cuadros de amor y humor, al fresco, Madrid, Cátedra.

- 2006 b. «Teatro breve en los inicios del siglo xxi: Los «cuadros» de Alonso de Santos», en José Romera Castillo, ed. , y Francisco Gutiérrez Carbajo, cool., 2006, Las nuevas dramaturgias a comienzos del siglo $X X$, Madrid, Visor. 
Hedenreich, H. 1962. Figuren und komik in den spanischen «Entremeses» des goldenen Zeitalters. Munic: Ludwig-Maximilian Univ.

Hormigón, Juan Antonio. 1972. «Del Mirlo Blanco a los teatros independientes», Cuadernos Hispanoamericanos, 260 (1972), pp. 349355.

Huerta Calvo, Javier. 1996. El nuevo mundo de la risa. Estudios sobre el teatro breve y la comicidad en los siglos de oro, Palma de Mallorca, Olañeta.

- 1999 a. Antología del teatro breve español del siglo XVII, Madrid, Biblioteca Nueva.

- 1999 b, ed.,Teatro y Carnaval. En Cuadernos de Teatro Clásico, 12.

- 2000, ed., El gran mundo del teatro breve; $\mathrm{n}^{\circ}$ monográfico de Ínsula, 639-640.

- 2001. El teatro breve en la Edad de Oro, Madrid, Laberinto.

JACK, W. S. 1923. The early Entremés in Spain: the Rise of a Dramatic Form, Filadelfia, University of Pennsivalnia.

JAMESON, Francis. 1989 a. Documentos de cultura, documentos de barbarie, Madrid, Visor.

- 1989 b. Ensayos sobre el postmodernismo, Buenos Aires, Imago Mundi.

Lotman, Iuri. 1978. Estructura del texto artístico, Madrid, Istmo.

Llerena, Joseph María. 1980. «Los agarrotados de don Pío», en Mundo Diario, 18-IV-1980, incluido en Pío Baroja, El horroroso crimen de Peñaranda del Campo, Madrid, Vox, 1980, pp. 35-36.

Llovet, Enrique. 1979. «Baroja con amor», El Pais, 21-I-1979; incluido en Pío Baroja, El horroroso crimen de Peñaranda del Campo, Madrid, Vox, 1980, pp. 33-34.

Medina Vicario, Miguel. 1994. Los géneros dramáticos en la obra teatral de José Luis Alonso de Santos, Madrid, Ediciones Libertarias y AAT.

MonLEÓn, José. 1980. «Baroja y el teatro». En Pío Baroja, El horroroso crimen de Peñaranda del Campo, Madrid, Vox, 1980, pp. 9-22.

Oliva, César. 1989. El teatro desde 1936, Madrid, Alhambra.

- 2001. Int. a Yonquis y yanquis. Salvajes, de José Luis Alonso de Santos, Madrid, Castalia.

Olivera Santos, María Teresa. (1998. (ed.). José Luis Alonso de Santos, iViva el Duque, nuestro dueño! y La estanquera de Vallecas, Madrid, Alhambra.

Peral Vega, Emilio. J. 2001. Formas del teatro breve español en el siglo XX (1892-1939), Madrid, FUE. 
Pérez, L. C. (ed.) Francisco Ortiz, Apología en defensa de las comedias que se representan en España. Valencia: Estudios de Hispanófila, 1977, 53-54, Apud. Granja, A. de la (1994: 164).

Pérez Priego, Miguel Ángel. 2004. El teatro en el Renacimiento, Madrid, Laberinto.

Piñero, Margarita. 2001. Int. a Dígaselo con Valium, de José Luis Alonso de Santos, El Puerto de Santa María, Fundación Pedro Muñoz Seca y Hogar Sur.

- 2004. Introducción a El Buscón, versión teatral de J.L. Alonso de Santos a partir de «Historia de la vida del Buscón llamado don Pablos», de Francisco de Quevedo, Madrid, RESAD/ Fundamentos.

- 2005. La creación teatral en José Luis Alonso de Santos, Madrid, Fundamentos /RESAD.

PlazA, Sixto. 1983. «Pío Baroja y su original aportación al teatro musical español», Cuadernos de ALDEEU, 1 (enero, 1983), pp. 101-107. Primer Acto, 143, abril 1972. Monográfico sobre el teatro de Baroja.

RAFFI-BÉroud, C.M.H. (2001). «Teatro musical o político breve: siglo XVIII-principio del siglo XIX». En VERSTEEG, M. (dir.) (2001) En torno al teatro breve, Ámsterdam-Atlanta, Rodopi, 65-74.

Rey Faraldos, Gloria. 1985. «Pío Baroja y El Mirlo Blanco», Revista de Literatura, XLVII, 93 (1985), pp. 117-127.

Ríos Carratalá, Juan A. 2005. La memoria del humor, Alicante, Publicaciones de la Universidad de Alicante.

Rodríguez, Evangelina - TorderA, Antonio (eds.) 1990. Calderón de la Barca, Entremeses, jácaras y mojigangas, Madrid, Castalia.

Rodríguez Adrados, Francisco. 1983. Fiesta, comedia y tragedia, Madrid. Alianza.

Romera Castillo, José. 2002. Pról. a Mis versiones de Plauto. Anfitrión, La dulce Cásina y Miles gloriosus, de José Luis Alonso de Santos, Madrid, UNED.

Rubio Jiménez, Jesús.1990. «Valle-Inclán y los teatros independientes de su tiempo», Letras de Deusto, 48 (sept.-dic., 1990), pp. 49-72.

- 1993. El teatro poético en España. Del Modernismo a las Vanguardias, Murcia, Universidad de Murcia

- 1998. Int. a Pío Baroja, ¡Adiós a la bohemia!, Arlequín, mancebo de botica y El horroroso crimen de Peñaranda del Campo, Madrid, Biblioteca Nueva-Fundación Argentaria, pp. 9-75.

Schoentues, Pierre. 2003. La poética de la ironía, Madrid, Cátedra.

Serrano, Virtudes. 2004. (ed.) Teatro breve entre dos siglos, Madrid, Cátedra. 
Ubersfeld, Anne. 1981. L'école du spectateur. Lire le théâtre, 2. París, Ed. Sociales.

- (1989). Semiótica teatral, Madrid, Cátedra.

VERSTEEG, M. 2000. De fusiladores y morcilleros. El discurso cómico del género chico, Amsterdam-Atlanta, Rodopi.

- (dir.).2001. En torno al teatro breve, Amsterdam-Atlanta, Rodopi.

Vila Selma, José. 1956. «Pío Baroja. Crítico teatral», Teatro 20 (sept.-dic., 1956), pp.6-7.

Ynduráin, Domingo. 1978. «Pío Baroja, al fin en los escenarios», El País (20-VIII-1978), recogido en Pío Baroja, El horroroso crimen de Peñaranda del Campo, Madrid, Vox, 1980, pp. 23-27 